\title{
Timing Analysis of V404 Cyg during Its Brightest Outburst with INTEGRAL/SPI
}

\author{
J. Rodi ${ }^{1,2}$, E. Jourdain ${ }^{1,2}$, and J. P. Roques ${ }^{1,2}$ \\ ${ }^{1}$ Université de Toulouse; UPS-OMP; IRAP; Toulouse, France \\ ${ }^{2}$ CNRS; IRAP; 9 Av. Colonel Roche, BP 44346, F-31028 Toulouse cedex 4, France
}

\begin{abstract}
The outburst of V404 Cyg during the summer of 2015 reached unparalleled intensities at $\mathrm{X}$-ray and soft gamma-ray energies with fluxes $>50 \mathrm{Crab}$ in the $20-50 \mathrm{keV}$ energy band. To date, studies in the hard X-ray/soft gamma-ray energy domain have focused primarily on the energy spectra. In this work, timing analysis has been performed with INTEGRAL/SPI data in the $20-300 \mathrm{keV}$ energy range for INTEGRAL revolution 1557, which corresponds to the brightest flare of V404 Cyg (on June, 26). The power spectra are fit with broken power-law and multi-Lorentzian models and compared with previously reported results of V404 Cyg flaring activity from 1989 and 2015. Also, we took advantage of the good signal-to-noise ratio obtained above $70 \mathrm{keV}$ to quantify the timing/fast-variability properties of the source as a function of energy. We then point out similarities of V404 Cyg with the black hole transient V4641 Sgr. Like V4641 Sgr, we found the power spectra of V404 Cyg during high flux periods did not possess the expected flat-top feature typically seen in a hard spectral state. Interpretations are proposed in the framework of the fluctuating-propagation model to explain the observed properties.
\end{abstract}

Subject headings: X-rays: general — X-rays: binaries — stars: black holes — stars: individual (V404 Cygni)

\section{Introduction}

On 2015 June 15 at 18:31:38 UTC (MJD 57188.772), the Swift/Burst Alert Telescope (BAT) triggered on the black hole $(\mathrm{BH})$ transient V404 Cyg (GS 2023 + 338) (Barthelmy et al. 2015), which began a $\sim 1$ month-long period of strong activity with unprecedented coverage at all wavelengths. In particular in the hard X-rays, intensities reached values $>50 \mathrm{Crab}$ in the $20-50 \mathrm{keV}$ band (Jourdain et al. 2017). So far, most analyses at hard X-ray/soft gamma-ray energies have focused primarily on the energy spectra, which have found the source spectrum well-described by a Comptonization model, plus a second component to take into account the high energy tail (Roques et al. 2015, Natalucci et al. 2015, Jenke et al. 2016, Jourdain et al. 2017). Together with an absent or moderate thermal (disk) emission (see for instance Radhika et al. (2016)), this suggests the source to be mainly in Hard (or Intermediate Hard) State.
However, in these works, Comptonization models have found best-fit parameters difficult to explain, reinforcing the atypical behavior of V404 Cyg mentioned since its 1989 outburst (Oosterbroek et al. 1997). The reported seed photon temperatures are $\sim 5-7 \mathrm{keV}$ (Roques et al. 2015, Natalucci et al. 2015, Jenke et al. |2016, Jourdain et al. 2017), which are significantly hotter than the $\sim 1 \mathrm{keV}$ that can be produced by an accretion disk. It could suggest that the seed photons are due to another process such as bremsstrahlung or synchrotron emission (Markoff et al. 2005: Done et al. 2007). On the other hand, absorption values of $\sim 1-$ a few $10^{24} \mathrm{~cm}^{-2}$ (Jenke et al. 2016, Jourdain et al. 2017: Motta et al. 2017) and/or a very strong reflection component may provide a good description of the data, confirming the complex variable geometry reported during the previous outburst (Oosterbroek et al. 1997; Zycki et al. 1999). 
Other properties of the systems, such as accretion geometry and the dynamics of the Comptonizing medium, can be studied through timing analysis (Kazanas et al. 1997; Reig et al. 2003. Giannios et al. 2004). While many instructive results are available in the X-ray domain, results in the hard X-ray regime are few, where photons are much less numerous. Such results are particularly important to investigate the emission mechanism at work above $20 \mathrm{keV}$.

In this work, we report on timing analysis of INTEGRAL/SPI data in the $20-300 \mathrm{keV}$ energy range, around the brightest period of the V404 Cyg outburst. First, we describe the observations along with the analysis method. Next, we present our power spectra as well as time lags between low and high energy bands. Finally, we discuss our results in context of other results from V404 Cyg and the BH transient V4641 Sgr.

\section{Observations and Data Analysis}

INTEGRAL/SPI (Vedrenne et al. 2003; Roques et al. 2003) observations of V404 Cyg during the outburst maximum occurred during revolution 1557, which spanned 2015 June 26 01:32 - 27 23:47 UTC (MJD 57199.064 - 57200.991). During this revolution, V404 Cyg underwent two major flares, as shown in Fig. 1. The light curves cover the $20-50 \mathrm{keV}$ (top) and $50-300 \mathrm{keV}$ (bottom) energy bands with $300 \mathrm{~s}$ integration for each point.

A study of the temporal behavior of V404 Cyg was conducted using four time periods during which the source was extremely bright to maximize the signal-to-noise ratio (SNR) and to minimize any contamination from Cyg X-1, which is in the SPI field of view. However, the loss of telemetry packets during the brightest times of the second flare, even though moderate $(\sim 10-15 \%)$, made timing analysis difficult thus data from this time span were not analyzed. The data sets studied in this work are plotted in red, green, blue, and purple, respectively, with the start and stop times listed in Table 1. The first period (red) spans the majority of the first flare, beginning when the $20-50 \mathrm{keV}$ flux exceeds $\sim 5$ Crab after a shortlived drop and lasting until the flare begins its smooth decay to a "low" flux state. The second period (green) covers the rise of the second flare while the third period (blue) consists of the de- cay of the second flare. The last period (purple) corresponds to the tail of the flare and is contemporaneous with Fermi/GBM observations used by Jenke et al. (2016). It marks the end of the period of exceptional activity.

For each period, power density spectra (PDS) were made in three energy ranges. A broad $20-300 \mathrm{keV}$ energy range was studied first to investigate the general shape of the power spectra. Then two narrower energy bands, 20 - 50 $\mathrm{keV}$ and $69-300 \mathrm{keV}$, were analyzed to study the behavior as a function of energy. Because of intense background lines (Weidenspointner et al. 2003 ), the $50-69 \mathrm{keV}$ and $190-215 \mathrm{keV}$ energy ranges were excluded in constructing the high energy band. The data from individual germanium detectors were summed together for getting the total number of counts incident on the detector plane. In constructing a single PDS, 100-s long segments were used, and the SPI data, which have a time resolution of $102.4 \mu \mathrm{s}$ (Vedrenne et al. 2003). The data were rebinned to have a Nyquist frequency of $81 \mathrm{~Hz}\left(f_{N y q}=1 /(2 \Delta t)\right.$, where $\Delta t$ is the time bin width; $\Delta t=614.4 \mu s$ here), which allowed for studying frequencies from $0.01-81 \mathrm{~Hz}$. Power spectra analysis was performed using the Interactive Spectral Interpretation System (ISIS) (Houck \& Denicola 2000).

PDS of empty field or background observations will still result in a nonzero average value because of statistical variability. Using the Leahy normalization (Leahy et al. 1983), the mean value of the PDS should be 2 in the case of only a background signal. To verify that the SPI data behave as expected, data from $\sim 70000 \mathrm{~s}$ of observations from revolution 1549 were used as an empty field observation (centered on $\alpha=12: 30: 00, \delta=$ +10 : 00 : 00). Fig. 2 shows the Leahy normalized PDS from the empty field period in the 20 - $300 \mathrm{keV}$ energy range. The PDS displays a fairly constant power with small scatter from $\sim 1-81 \mathrm{~Hz}$. Below $\sim 1 \mathrm{~Hz}$, the scatter increases, though the sizes of the error bars increase as well. A fit to a constant value finds an average power of the PDS of $2.0099 \pm 0.0009$ with $\chi^{2} / \nu=0.889$ $(\nu=35)$, which is close to the expected value. As the SPI data show values close to 2 for the total energy range as well as for the two narrower energy bands, the theoretical value of 2 was used to remove the background component in converting 
Table 1: INTEGRAL/SPI observations of V404 Cyg during Revolution 1557 used in this work

\begin{tabular}{cccc}
\hline \hline Period & & Time & Time \\
& & $($ MJD $)$ & $($ UTC) \\
\hline 1 & Flare 1 & $57199.110-57199.250$ & 2015 June 26 02:38:24-06:00:00 \\
2 & Flare 2 Rise & $57199.470-57199.510$ & 2015 June 26 11:16:48 - 12:14:24 \\
3 & Flare 2 Decay & $57199.845-57199.860$ & 2015 June 26 20:16:48 - 20:38:24 \\
4 & Flare 2 Tail & $57200.082-57200.148$ & 2015 June 27 01:58:04-03:33:07 \\
\hline \hline
\end{tabular}
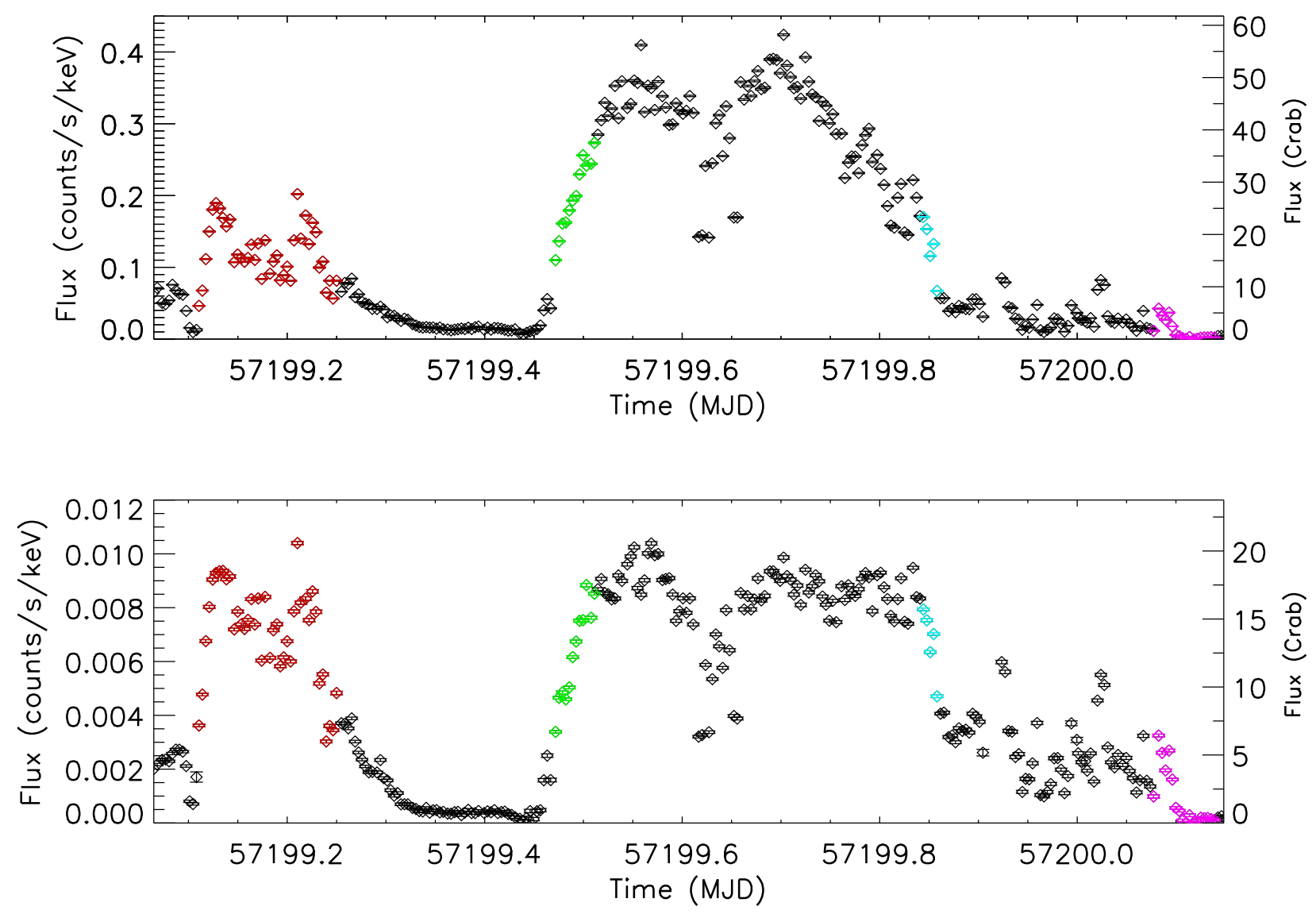

Fig. 1.- Top: INTEGRAL/SPI light curve of V404 Cyg in $20-50 \mathrm{keV}$ band spanning MJD $57199.064-$ 57200.150. Points are $300 \mathrm{~s}$ integration time. The red, green, and blue periods are high SNR times that do not suffer from telemetry loss. The purple period allows for the study of a low flux period and is also contemporaneous with Fermi/GBM observations. Bottom: Light curve of V404 Cyg in 50-300 keV energy band with the observation periods denoted with the same colors as above. 
the PDS to rms normalization (Belloni \& Hasinger 1990; Miyamoto et al. 1991).

\section{Results}

\subsection{Power Spectra Density Fits}

Power spectra can often be described by multiple components that are generally classified as either band-limited noise (BLN), which are broad features, or quasi-periodic oscillations (QPOs), which are narrow features (Belloni et al. 2002).

Initially, the V404 Cyg data were fit with a two Lorentzian model, but large positive features in the residuals were often found, suggesting the existence of a third component except for observation period 4 in which the SNR is low. The presence of three broad features is consistent with Belloni et al. (2002), who found similar results in nine of the 10 compact systems they studied, as well as with the analysis from the 1989 outburst of V404 Cyg by Oosterbroek et al. (1997). All the Lorentzians are zero-centered and no QPOs were detected in this work.

Adopting the notation from Belloni et al. (2002), the lowest frequency BLN component, $L_{b}$, corresponds to the "break" in the power spectrum at frequency $\nu_{b}$. The other two BLN components are fit by the Lorentzians $L_{l}$ and $L_{u}$ with frequencies $\nu_{l}$ and $\nu_{u}$ to cover the "lower" and "upper"

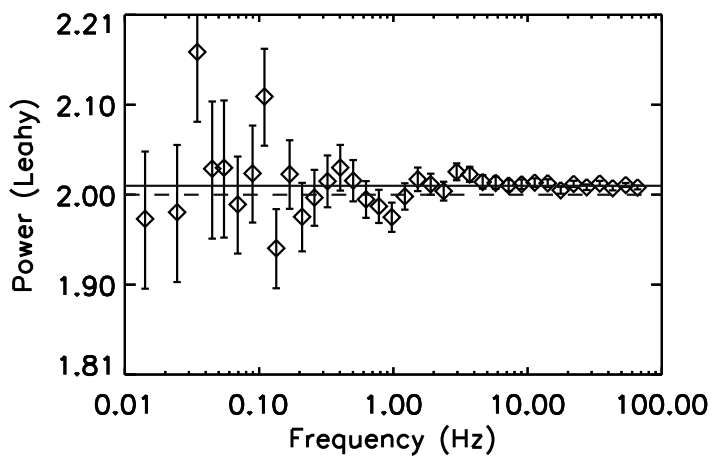

Fig. 2.- PDS of $\sim 70000$ s of empty field observations from revolution 1549 for the $20-300 \mathrm{keV}$ energy range. Solid line plotted at average power at 2.0099; Dashed line at power of 2 . parts of the power spectrum.

The power spectra for the four observation periods are plotted in Fig. 3 with the best-fit model overplotted and the residuals plotted below. For the first three observation periods, the power spectra are significantly detected up to $\sim 5-10 \mathrm{~Hz}$. The flux level of V404 Cyg during observation period 4 is drastically lower than for the others so a lower SNR is expected. The source signal is significantly detected out to only $\sim 0.5 \mathrm{~Hz}$ while presenting the highest power at low frequencies.

The results of the fits to each power spectrum are listed in Table 2, which includes in the top panel the $\nu_{\max }$ and rms for the three (or two) Lorentzians along with the total $\mathrm{rms}$ and $\chi^{2} / \nu$ values. The errors listed are the $1-\sigma$ values.

The peak frequencies of the SPI power spectra have a narrow range for each of the Lorentzians: $\sim 0.012-0.034 \mathrm{~Hz}, \sim 0.2-0.6 \mathrm{~Hz}$, and $\sim 1.8-2.8$ $\mathrm{Hz}$, for $\nu_{b}, \nu_{l}$, and $\nu_{u}$, respectively. The break Lorentzian always dominates the total rms, spanning $\sim 11.5-26 \%$, while $\mathrm{rms}_{l}$ and $\mathrm{rms}_{u}$ remain between $\sim 6-11 \%$.

Considering the energy domain accessible in our data, we were able to investigate the evolution of the rms values in the hard X-ray domain. We thus built power spectra for each of the four time periods in two energy bands: $20-50 \mathrm{keV}$ and $69-300$ $\mathrm{keV}$. We fixed the $\nu_{\max }$ frequencies to the $20-300$ $\mathrm{keV}$ ones (Table 2) and determined the rms values. Results are displayed in Fig. 4, where panels (a), (b), and (c) correspond to $\mathrm{L}_{b}, \mathrm{~L}_{l}$, and $\mathrm{L}_{u}$, respectively, while black diamonds and red triangles stand for $20-50 \mathrm{keV}$ and $69-300 \mathrm{keV}$ bands. For each Lorentzian, the rms values follow a similar pattern, with an upward trend between periods $2-4$ (i.e. during the second flare). However, the evolution with energy is different for each period: in periods 1 and 3 , the low energy values are larger by a factor of $\sim 1.5$ for $\mathrm{rms}_{b}$ and $\mathrm{rms}_{l}$. For $\mathrm{rms}_{u}$, the ratio decreases to $\sim 1.1$ for period 1 while for period 3 , the ratio is approximately 1 . For period 2, the ratio decreases with increasing frequency from $\sim 1.2$ to $\sim 0.9$ to $\sim 0.6$. For period 4 , the rates are close to 1 with consistent values between the low and high energy rms values for both Lorentzians.

The power spectra were also fit using broken power-law models for comparison with other re- 

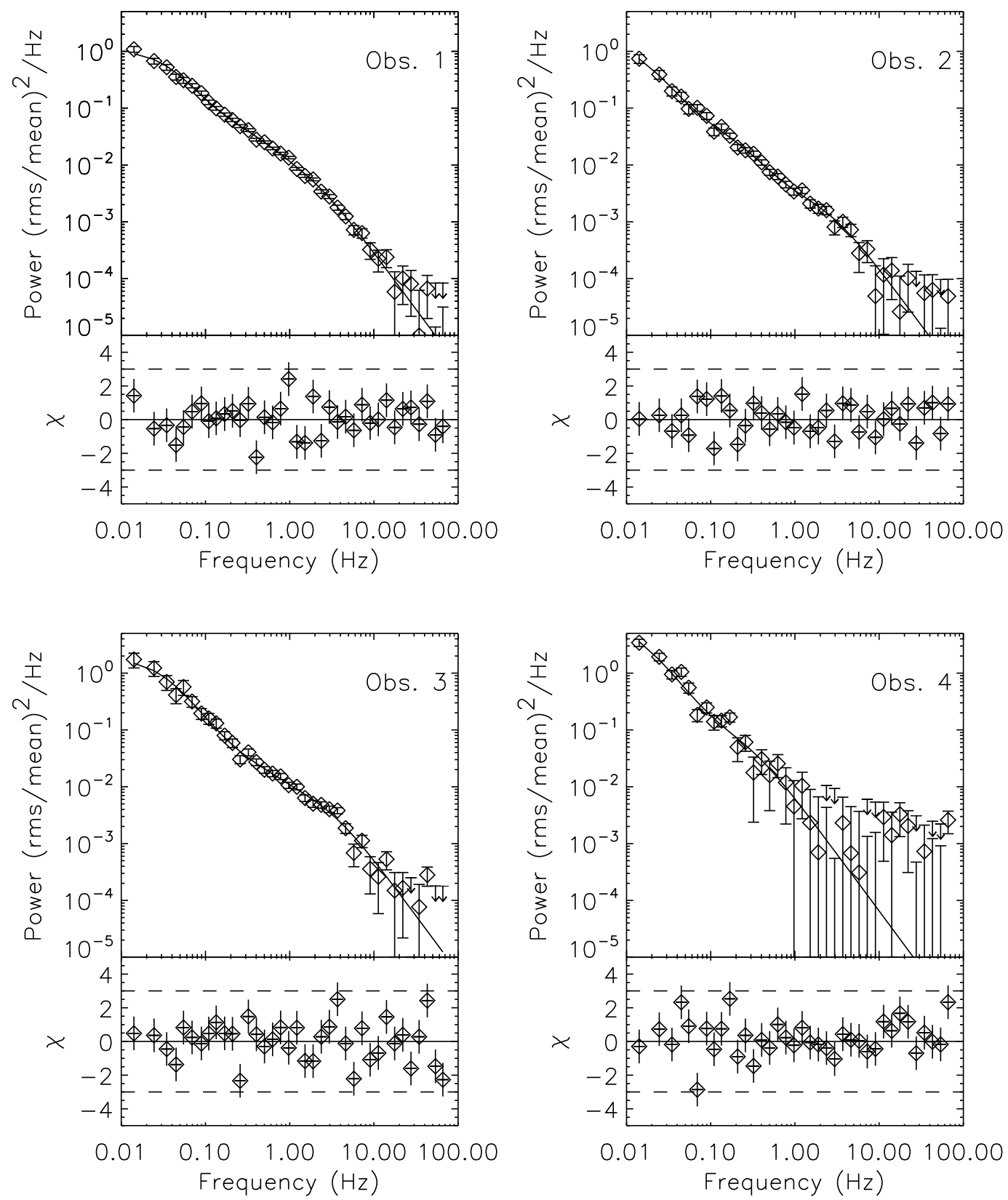

Fig. 3.- The $20-300 \mathrm{keV}$ power spectra of the four observation periods with two or three Lorentzian fit models overplotted. The corresponding residuals are plotted below. The solid line denotes $0 \sigma$, and the dashed lines denote $\pm 3 \sigma$. See Table 2 for the model best-fit parameters. 
sults or model predictions. (See Sec. 4.3 and Sec. 4.4.1.) The fit parameters are listed in the bottom panel Table 2 with observation 4 requiring only a single power law. For observations 1, 2 and 4 , the $\chi^{2} / \nu$ values are similar to those obtained with multi-Lorentzian models. Observation 3 residuals suggested the need for a low frequency $(<\sim 0.1 \mathrm{~Hz})$ component. Adding a third powerlaw results in a slope of 1.48 below $0.26 \mathrm{~Hz}$ while the middle power law hardens to $\sim 1$, the highfrequency power-law softens to roughly 2.1 and the $\chi^{2} / \nu$ value decreases to 1.43 . If a Lorentzian is included instead, a peak frequency of $0.023_{-0.006}^{+0.008} \mathrm{~Hz}$ is found, which is consistent with $L_{b}$ in the three Lorentzian model. The final $\chi^{2} / \nu$ value reduces to 1.46. We note that the inclusion of a third powerlaw or a Lorentzian results in a better $\chi^{2} / \nu$ value than the multi-Lorentzian model.

\subsection{Time Lags}

In addition to generating power spectra, the time lags between the $20-50 \mathrm{keV}$ and $69-300 \mathrm{keV}$ energy bands were determined. Only the first period possessed high enough statistics to make any conclusive statements. Fig. 5 shows the time lag with positive lags indicating that the hard photons lag the soft ones. The time lags follow a power law over the frequency range, spanning $\sim 10^{-3}-1 \mathrm{~s}$, though above $\sim 3 \mathrm{~Hz}$, the lag values are poorly constrained. The best-fit power law to the data has an index of $\Gamma=0.79_{-0.07}^{+0.07}\left(\chi^{2} / \nu=1.016\right)$.

\section{Discussion}

\subsection{SPI results}

Broken power-law and multi-Lorentzian models provide a similarly good description of the data in terms of $\chi^{2} / \nu$ values. In both cases, the obtained parameters point out some evolution with time.

Periods 1 and 3 are globally alike. Lorentzian and broken power-law parameters are close, except that an excess of power at low frequencies requires one additional power-law for period 3. For both periods, rms values are significantly higher at low energy and low frequency. To relate timing properties and spectral ones, we note that period 3 corresponds to the end of the decay of the second flare, during which the emission is observed to recover the same hardness as period 1 , through a
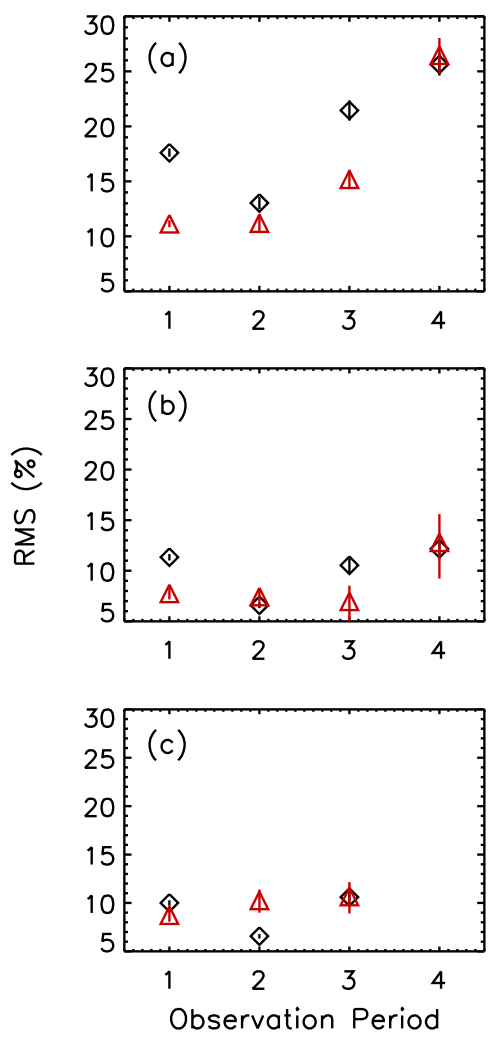

Fig. 4.- RMS frequency values for $\mathrm{L}_{b}, \mathrm{~L}_{l}$, and $\mathrm{L}_{u}$ in panels (a), (b), and (c), respectively. The SPI $20-50 \mathrm{keV}$ results are plotted as black diamonds, and the $69-300 \mathrm{keV}$ results are plotted in red triangles.

decrease of the low energy emission $(20-50 \mathrm{keV}$; see Fig. 1 or Jourdain et al. (2017)).

On the other hand, during period 2, (along the rise phase of the second flare), the spectral evolution is driven by the increase of the low energy part (see Fig. 1). In the time domain, the variability is lower, particularly at low frequency (low $\mathrm{rms}_{t o t}$ and $\mathrm{rms}_{b}$ or steeper $\left.\Gamma_{1}\right)$. Fig. 4 shows that this decrease is mainly due to low energy: while the rms values are comparable to those of period 1 for the $69-300 \mathrm{keV}$ energy band, they are notably smaller in the $20-50 \mathrm{keV}$ band.

Finally, the 4th period behaves quite differently from the others. The lower SNR could explain the absence of signal above $1 \mathrm{~Hz}$ in the PDS and a simple power-law describing the PDS spectrum. 
Table 2: Power Spectra Fits

\begin{tabular}{|c|c|c|c|c|}
\hline Obs. period & 1 & 2 & 3 & 4 \\
\hline \multicolumn{5}{|c|}{ Multi-Lorentzian } \\
\hline$\nu_{b}(\mathrm{~Hz})$ & $0.034_{-0.007}^{+0.005}$ & $0.012_{-0.004}^{+0.005}$ & $0.029_{-0.006}^{+0.008}$ & $0.012_{-0.003}^{+0.003}$ \\
\hline $\mathrm{rms}_{b}(\%)$ & $15.0_{-0.6}^{+0.4}$ & $11.5_{-1.0}^{+1.7}$ & $18.5_{-1.3}^{+1.4}$ & $25.9_{-1.9}^{+2.8}$ \\
\hline$\nu_{l}(\mathrm{~Hz})$ & $0.4_{-0.2}^{+0.2}$ & $0.21_{-0.05}^{+0.06}$ & $0.6_{-0.3}^{+0.3}$ & $0.3_{-0.1}^{+0.2}$ \\
\hline $\mathrm{rms}_{l}(\%)$ & $9.5_{-1.3}^{+1.3}$ & $6.5_{-0.4}^{+0.4}$ & $7.7_{-1.7}^{+1.4}$ & $10.9_{-1.4}^{+1.2}$ \\
\hline$\nu_{u}(\mathrm{~Hz})$ & $1.8_{-0.5}^{+0.9}$ & $2.4_{-0.5}^{+0.7}$ & $2.8_{-0.5}^{+0.8}$ & - \\
\hline $\mathrm{rms}_{u}(\%)$ & $8.8_{-2.1}^{+1.7}$ & $5.9_{-0.4}^{+0.3}$ & $10.2_{-1.5}^{+1.0}$ & - \\
\hline $\mathrm{rms}_{t o t}(\%)$ & $19.8_{-1.9}^{+1.7}$ & $14.5_{-1.2}^{+1.7}$ & $22.6_{-2.3}^{+2.1}$ & $28.1_{-2.3}^{+3.0}$ \\
\hline$\chi^{2} / \nu$ & 1.04 & 0.94 & 1.60 & 1.27 \\
\hline \multicolumn{5}{|c|}{ Power Law } \\
\hline$\Gamma_{1}$ & $-1.11_{-0.01}^{+0.01}$ & $-1.20_{-0.02}^{+0.03}$ & $-1.09_{-0.04}^{+0.03}$ & $-1.50_{-0.06}^{+0.07}$ \\
\hline$\nu_{B}(\mathrm{~Hz})$ & $1.9_{-0.2}^{+0.01}$ & $4.7_{-1.4}^{+-2} .4$ & $3.7_{-0.2}^{+0.0}$ & -0.06 \\
\hline$\Gamma_{2}$ & $-1.71_{-0.07}^{+0.07}$ & $-1.78_{-0.8}^{+0.4}$ & $-1.8_{-0.3}^{+0.2}$ & - \\
\hline$\chi^{2} / \nu$ & 1.12 & 0.99 & $2.07^{\mathrm{a}}$ & 1.30 \\
\hline
\end{tabular}

${ }^{a} \mathrm{~A}$ better model is proposed in Sec. 3.1.

However, its (low frequency) slope of 1.5 is similar to that of the component added for describing the low frequency part of period 3. It can also be seen as an intermediate value between the low and high frequency indices found in periods 1 and 2. In addition, because of the comparatively large $\mathrm{rms}_{b}$, the $\mathrm{rms}_{\text {tot }}$ for period 4 is the highest (28.1\%) with respect to the other periods $(14.5-22.6 \%)$. Moreover, as already mentioned, the rms values are unchanged from 20 to $300 \mathrm{keV}$. It should be noted that this period corresponds to a spectrally more stable state, where the Compton emission is decreasing while the hard tail component takes over, producing a particularly hard spectral shape (Jourdain et al. 2017).

\subsection{Comparison with Other Observations}

Due to the unprecedented intensities at $\mathrm{X} / \mathrm{hard}$ $\mathrm{X}$-ray energies and the duration of the outburst, other Fourier-based studies have been performed on V404 Cyg data. Observations from the Swift/X-Ray Telescope (XRT) during MJD 57191 show a weak QPO detection at $1.7 \mathrm{~Hz}$ in the $0.3-10 \mathrm{keV}$ band during a low flux pe- riod (Motta et al. 2015). Huppenkothen et al. (2017) have reported detecting several QPOs at frequencies of $18 \mathrm{mHz}, 73 \mathrm{mHz}, 136 \mathrm{mHz}$, and $1.03 \mathrm{~Hz}$ with Fermi/GBM, Swift/XRT, and the Chandra/Advanced CCD Imaging Spectrometer (ACIS). Conversely, Jenke et al. (2016) analyzed the Fermi/Gamma-ray Burst Monitor (GBM) data from MJD 57188 - 57200 and were able to fit the data with two or three broad Lorentzians and do not report any QPOs. Also, Radhika et al. (2016) reported no detection of QPOs during 2015 June observations with Swift/XRT. Similar to the Jenke et al. (2016) GBM results, we were able to describe the SPI PDS with two or three broad Lorentzians with no QPOs detected.

Concurrent observations between INTEGRAL and GBM allowed for a comparison between the two instruments. GBM analysis reported a contemporaneous observation with period 4 (MJD $57200.082-57200.148$ ). Jenke et al. (2016) used 8-100 keV data, extending to lower energies than the SPI data. Data sets from both instruments required only two BLN components. For the $\nu_{b}$ values, Jenke et al. (2016) found a best-fit value of 


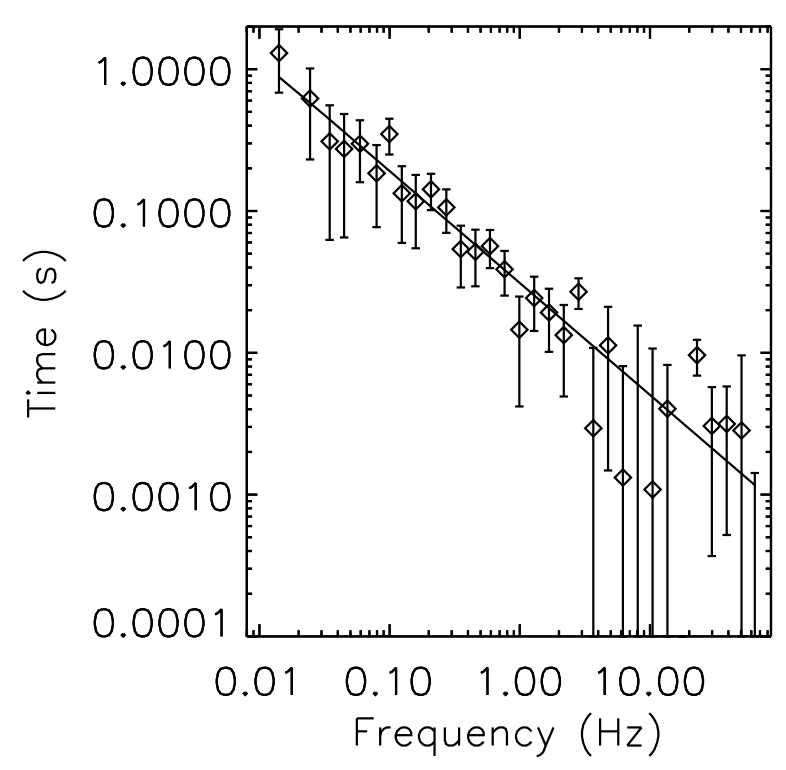

Fig. 5. - Time lags between the $20-50 \mathrm{keV}$ and 69-300 keV energy bands from observation period 1.

$(1.6 \pm 0.5) \times 10^{-2} \mathrm{~Hz}$ compared to $(1.2 \pm 0.3) \times 10^{-2}$ $\mathrm{Hz}$ in the SPI $20-300 \mathrm{keV}$ results and thus the results are marginally consistent. The $\nu_{l}$ values are significantly different with Jenke et al. (2016) finding $0.61 \pm 0.1 \mathrm{~Hz}$ while this analysis found $0.3 \pm 0.1$ $\mathrm{Hz}$. A fit to the SPI $20-300 \mathrm{keV}$ power spectrum with the frequencies fixed to the GBM values finds a $\chi^{2} / \nu$ of 1.29 , only slightly higher than the 1.27 found when both frequencies are left free, but the $\chi^{2}$ is larger by 3.01. However, the small disagreement is potentially due to the different energy ranges analyzed from the two instruments.

\subsection{Outburst vs 2015 Outburst}

Syunyaev et al. (1991) report on observations of V404 Cyg (=GS 2023+338) 1989 outburst by the Roentgen observatory. They were able to perform a timing analysis with the HEXE data $(15-200$ $\mathrm{keV}$ ) when the source flux had decreased to $\mathrm{a} \sim 1$ Crab level. They found that the power spectrum was dominated by a strong low-frequency noise between $0.01-5 \mathrm{~Hz}$, approximated by a power-law with a slope $\sim f^{-1}$ in the energy range $20-100$ $\mathrm{keV}$. Moreover, the same analysis performed on the $20-35 \mathrm{keV}$ and $65-180 \mathrm{keV}$ energy band showed similar power spectra in shape and amplitude.

These results can be compared with our period 4 data, where the average flux level $(\sim 2$ Crab in the $20-300 \mathrm{keV}$ band) is close to the Syunyaev et al. (1991) observations. During this relatively low flux period, the SPI power spectrum is also dominated by low-frequency variability and its rms values remain unchanged with energy. However, the low-frequency slope is significantly steeper with a slope of -1.5 .

Power spectra analysis was also performed for the 1989 outburst of V404 Cyg by Oosterbroek et al. (1997), using Ginga data in the $1.2-36.8$ keV energy range. Oosterbroek et al. (1997) made power spectra covering $\sim 0.004-512 \mathrm{~Hz}$ and $\sim 0.004-8 \mathrm{~Hz}$ based on the two available timeresolution data. For both data sets, the model was comprised of two zero-centered Lorentzians to fit the low frequency regime and one non-zerocentered Lorentzian for the higher frequency portion of the power spectrum. The results consist of a large variety of best-fit parameters, even if the authors concluded that the shape of the power spectrum does not change significantly. Our peak frequency and rms values are commensurable with the ranges observed in the Ginga data. On the other hand, we note that many of the Ginga power spectra present a flattening below $\sim 0.1 \mathrm{~Hz}$, we fail to detect in our data sets. If we ascribe that to the different energy ranges used in the analyses, it could imply that different physical mechanisms are at work, and behave dissimilarly.

Considering the rms fractions, they range, in the Ginga data sets, between 20 and 40\% (0.01-8 $\mathrm{Hz}$ ) slightly higher than the values found in our analysis. Also, they appear stable above $10 \mathrm{keV}$ up to $\sim 30 \mathrm{keV}$, in contrast with the significant decrease observed above $50 \mathrm{keV}$ in the SPI data set for periods 1 and 3. Here too, it may be due to the energy domain or to the huge diversity and variability of the source characteristics.

\subsection{Comparison with Other Sources}

\subsubsection{Power Spectra}

V404 Cyg reached uncommon luminosity levels and displayed an enormous flux variability at all wavelengths (e.g. Kimura et al. (2016) and references therein). The extreme levels of luminosity 
and variability were observed up to a few hundreds of keV (see Fig. 1), which is rarely observed due to low numbers of photons at these energies. The BH transient V4641 Sgr showed a similar behavior during its 1999 outburst, though such an event lasted for only a few hours. Its peak flux was $\sim 12$ Crab in the X-ray band and the energy spectrum, observed up to $\sim 200 \mathrm{keV}$, suggested V4641 Sgr was in the hard state (Wijnands \& van der Klis 2000; Revnivtsev et al. 2002). Interestingly, power spectra analysis of the source in the $2-22.1 \mathrm{keV}$ energy range shows no clear flat-top, though such a shape is generally expected for power spectra in the hard state. The power spectrum is well described by a broken power-law with $\Gamma_{1}=-1.03$, a break at $5.1 \mathrm{~Hz}$, and $\Gamma_{2}=-2.16$ (Wijnands \& van der Klis 2000) and high rms (up to 50\%), decreasing with energy (around 30\% between 20 and $60 \mathrm{keV}$ ). These values are very similar to those found during our period 3, when we include a third power-law to account for an additional lowfrequency component (see section 3.1). The V404 Cyg power spectra for periods 1 and 2 are also well described by a broken power-law shape with comparable low frequency slopes $\left(\Gamma_{1} \sim-1.1\right)$ but flatter high frequency slopes $\left(\Gamma_{2} \sim-1.7\right)$. The break frequencies are consistent or lower by a factor of a few with that of V4641 Sgr (Table 2 bottom panel), which could be important information on the source geometry.

Concerning the rms, the values obtained along the flare, between 15 and 30\%, are comparable to those obtained for Cyg X-1 in similar energy bands from SPI data (Cabanac et al. 2011) or Suzaku data (Torii et al. 2011). Moreover, the rms behavior with energy is changing in V404 Cyg. For comparison, observations of Cyg X-1 by Cabanac et al. (2011) found a decreasing rms with energy while Torii et al. (2011) found a constant rms value from 20 to $200 \mathrm{keV}$.

\subsubsection{Time Lags}

Since Miyamoto et al. (1988), it has been known that the hard state X-ray spectrum cannot be due to a uniform Comptonizing medium, which predicts a roughly constant time lag. Instead observations find a power-law dependence with longer time lags at lower frequencies. The time lags range from milliseconds to a few seconds. The slope of $\Gamma=0.79$ seen in V404 Cyg is consistent with the $\nu^{-0.8}$ that has been found at hard X-rays for Cyg X-1 (Crary et al. 1998) and GRO J0422+32 (Grove et al. 1998). A study of many Cyg X-1 observations at lower energy (Pottschmidt et al. 2000) found also a similar trend, except during state transitions, implying that the lag behavior is independent of the source state.

\subsection{Physical Interpretation}

BLNs have been modeled as the result of shotnoise $^{1}$ processes resulting from such processes as magnetic flares or infalling blobs (see van der Klis (2006) and references within). Other proposed models involved self-organized critical state processes in which the accretion disk evolves to and remains in an organized state independent of the initial conditions (Mineshige et al. 1994). However, Uttley et al. (2005) deduced, from the linear rms-flux relation observed in many sources, that fluxes should follow a log-normal distribution (which was supported by Cyg X-1 data) meaning that the variability process is multiplicative. Consequently, additive shot-noise models (where shots on all time-scales are independent) and selforganized criticality models can be ruled out as they cannot reproduce these features (Uttley et al. 2005).

Another class of models, fluctuating-propagation models, have been developed (see for example Lyubarskii (1997)). They are multiplicative and thus are in agreement with a log-normal flux distribution. In such models, instabilities in the accretion flow at large distances from the source result in fluctuations in the region near the source where the hard X-rays are emitted, with fluctuations likely due to magnetorotational instabilities (Hawley \& Balbus 1991).

textbfIn the framework of the fluctuatingpropagation model, Churazov et al. (2001) considered an optically thin corona extending to large radii above and below the optically thick disk. They propose that instabilities occur at different (large) radii in the corona. The instabilities propagate towards the $\mathrm{BH}$ and modulate the mass accretion rate through a given radius. The product of the fluctuations from prior radii results in power-law shaped PDS over a wide range of

\footnotetext{
${ }^{1}$ Shot noise is a time-series model of random pulses (or shots), as suggested by Terrell \& Olsen (1970)
} 
frequencies (Lyubarskii 1997). Also, the flat-top features observed in the hard state are the result of damping by the truncated accretion disk. This damping occurs at the radius where the geometrically thin disk makes way for an optically thin accretion flow similar to the corona, typically below $\sim 1 \mathrm{~Hz}$.

The broken power-law shape seen in V404 Cyg periods $1-3$ and V4641 Sgr can be interpreted within the same framework. However, the power spectra for V404 Cyg and V4641 Sgr lack the typical flat-top features despite both sources being in a hard state. For the 1989 outburst of V404 Cyg (Tanaka \& Lewin 1995, Zycki et al. 1999) and the 1999 outburst of V4641 Sgr (Revnivtsev et al. 2002), results show evidence for a dense outflow produced from ejected material and suggesting disruptions of the disk (see Done et al. (2007) and references within). This unusual configuration could potentially arise from the sources being near or above the Eddington luminosity and reduce any damping effects from the disk. The extent to which the disk was disrupted would affect the frequency at which the flat-top begins.

With no flat-top detected in the SPI data down to $10^{-2} \mathrm{~Hz}$, a lower limit on the truncation radius can be estimated. Following the method employed in Gilfanov (2010) where the break frequency is equal to the inverse of the viscous time, a lower limit of the truncation radius is found to be $\sim$ $2500 r_{g}$. Interestingly, Zycki et al. (1999) estimate an unstable radius at $(5-8) \times 10^{10} \mathrm{~cm}(\sim 1200-$ $2300 r_{g}$ ), a value commensurable with our above estimation.

The fluctuating-propagation model can also explain the power-law dependence of the time lags. In such models, the temporal variability also corresponds to small variability in the local spectra. Assuming the local spectra emit like a power law with the slope decreasing with decreasing distance from the BH (Kotov et al. 2001; Arevalo \& Uttley 2006), the fluctuations at different distances in the accretion flow result in variability at different timescales being propagated towards the $\mathrm{BH}$. Moreover, recent works including Lense-Therring precession to account for QPOs have been able to model the power spectra of X-ray binaries using fluctuating-propagation models (Ingram \& Done 2011, 2012, Ingram \& van der Klis 2013).

\section{Conclusion}

We analyzed the $20-300 \mathrm{keV}$ power spectra for V404 Cyg during INTEGRAL revolution 1557 (MJD 57199.064 - 57200.991). The source intensity reached extreme levels up to a few hundred $\mathrm{keV}$, allowing timing studies in the hard X-ray domain. Power spectra from three high SNR periods showed three BLN features similar to reported behavior from Fermi/GBM observations during the same period (Jenke et al. 2016) and Ginga observations from the 1989 outburst (Oosterbroek et al. 1997). Like those works, no QPOs were detected in the SPI data.

The high SNR periods could also be modelled by broken power-laws. However, they do not show a clear flat-top typically seen in hard state power spectra. Similar behavior was reported for the 1989 outburst of V404 Cyg as well as for a strong outburst in another BH transient, V4641 Sgr. The fluctuating-propagation model provides a global interpretation of the observed properties. In this framework, the atypical power spectra (and light curves) for both sources are potentially due to accretion disk disruption related to the sources emitting near the Eddington luminosity.

A future perspective will be to explore the relation between spectral (disk) emission and timing properties as such an exceptional outburst conceals inestimable information about black hole physics and sub or super Eddington regimes.

\section{Acknowledgments}

The INTEGRAL SPI project has been completed under the responsibility and leadership of CNES. We are grateful to ASI, CEA, CNES, DLR, ESA, INTA, NASA and OSTC for support. JR acknowledges funding support CNES.

\section{REFERENCES}

Arevalo, P. \& Uttley, P. 2006, MNRAS, 367, 801

Barthelmy, S. D., Page, K. L., \& Palmer, D. M. 2015, GCN Circular 18716

Belloni, T. \& Hasinger, G. 1990, A\&A, 230, 103

Belloni, T., Psaltis, D., \& van der Klis, M. 2002, ApJ, 572, 392 
Cabanac, C., Roques, J.P., \& Jourdain, E. 2011, ApJ, 739, 758

Churazov, E., Gilfanov, M., \& Revnivtsev, M. 2001, MNRAS, 321, 759

Crary, D. J., Finger, M. H., Kouveliotou, C., van der Hooft, F. 1998, ApJ, 493, 71L

Done, C., Gierlinski, M., Kubota, A. 2007, A\&ARv, 15, 1

Giannios, D., Kylafis, N. D., \& Psaltis, D. 2004, A\&A, 425, 163

Gilfanov M., 2010, in Belloni T., ed., Lecture Notes in Physics, Vol. 794, The Jet Paradigm. Springer-Verlag, Berlin, p. 17

Grove, J. E., Strickman, M. S., Matz, S. M., Hua, X.-M. et al. 1998, ApJ, 502, 45L

Hawley, J. F. \& Balbus S. A. 1991, ApJ, 376, 223

Houck, J. C. \& Denicola, L. A. 2000, ASPC, 216, 591

Huppenkothen, D., Younes, G., Ingram. A., et al. 2017, ApJ, 834, 90

Ingram, A. \& Done, C. MNRAS, 2011, 415, 2323

Ingram, A. \& Done, C. MNRAS, 2012, 419, 2369

Ingram, A. \& van der Klis, M. 2013, MNRAS, 434, 1476

Jenke, P. A., Wilson-Hodge, C. A., Jeroen, H., et al. 2016, ApJ, 826, 37

Jourdain, E., Roques, J. P., \& Rodi, J. 2017, ApJ, 834,130

Kazanas, D., Hua, X., \& Titarchuk, L. 1997, ApJ, 480,735

Kimura, M., Isogai, K., Kato, T. et al. 2016, Nature, 529,54

Kotov, O., Churazov, E., \& Gilfanov, M. 2001, MNRAS, 327, 799

Leahy, D. A., Darbro, W., Elsner, et al. 1983, ApJ, 266,160

Lyubarskii, Y. E. 1997, MNRAS, 292, 679
Markoff, S., Nowak, M. A., \& Wilms, J. 2005, ApJ, 635,1203

Mineshige S., Takeuchi M., \& Nishimori H. 1994, ApJ, 435, L125

Miyamoto, S., Kitamoto, S., Mitsuda, K., \& Dotani 1988, Nature, 336, 450

Miyamoto, S., Kimura, K., Kitamoto, S., Dotani, T., \& Ebisawa, K. 1991, ApJ, 383, 784

Motta, S., Beardmore, A., Oates, S. et al. 2015, ATel, 7665, 1

Motta, S. E., Kajava, J. J. E., Sanchez-Fernandez, C., Giustini, M., Kuulkers, E. 2017, MNRAS, 468,981

Natalucci, L., Fiocchi, M., Bazzano, A. et al. 2015, ApJ, 813, 21

Oosterbroek, T., van der Klis, M., van Paradijs, J. et al. 1997, A\&A, 321, 776

Pottschmidt, K., Wilms, J., Nowak, M. A., et al. 2000, A\&A, 357, 17

Radhika, D., Nandi, A., Agrawal, V. K., \& Mandal, S. 2016, MNRAS, 462, 1834

Reig, P., Kylafis, N. D., \& Giannios, D. 2003, A\&A, 403, L15

Revnivtsev, M., Gilfanov, M., Churazov, E., \& Sunyaev, R. 2002, A\&A, 391, 1013

Roques, J. P., Schanne, S., von Kienlin, A. et al. 2003, A\&A, 411, L91

Roques, J. P., Jourdain, E. Bazzano, A., Fiocchi, M., Natalucci, L., \& Ubertini, Pietro 2015, ApJ, 813, 22L

Syunyaev, R. A., Kaniovskii, A. S., Efremov, V. A. et al., 1991, soviet astron. Lett., 17, 123

Tanaka, Y. \& Lewin, W. H. G., 1995, X-ray Binaries, eds. W. H. G. Lewin, J. van Paradijs, and W. P. J. van den Heuvel, Cambridge University Press

Terrell, J. \& Olsen, K. H., 1970, ApJ, vol 161, 399

Torii, T., Yamada, S., Makishima, K., et al. 2011, PASJ,63, 771 
Uttley, P., McHardy, I. M. \& Vaughan, S. 2005, MNRAS, 359, 345

van der Klis, M. 2006, in Compact stellar X-ray sources, ed. W. H. G. Lewin, \& M. van der Klis (Cambridge University Press) arXiv:astroph/0410551.

Vedrenne, G., Roques, J.-P., Schönfelder, V. et al. 2003, A\&A, 411, L63

Weidenspointner, G., Kiener, J., Gros, M., et al. 2003, A\&A, 411, 113L

Wijnands, R. \& van der Klis, M. 2000, ApJ, 528, 93L

Zycki, P. T., Done, C., \& Smith, D. A. 1999, MNRAS, 309, 561

This 2-column preprint was prepared with the AAS IATEX

macros v5.2. 